\title{
Infective Failure of Internal Fixation for Intertrochanteric Femoral Neck Fracture Presenting as Hip Joint Subluxation: A Case Report
}

\author{
Rohit Rambani ${ }^{\mathrm{a}, \mathrm{c}}$, Helen Ribee ${ }^{\mathrm{b}}$, Peter Bobak ${ }^{\mathrm{b}}$
}

\begin{abstract}
Complications following operative treatment of intertrochanteric fracture have previously been reported in the literature. Spontaneous subluxation following operating treatment of inter-trochanteric fracture has not been reported. We report a rare case of sponateous subluxation of the hip following a deep infection of the hip. An 86 years old female presented with sudden onset pain and difficulty in mobilization three months following a dynamic hip screw fixation. At surgery a deep wound infection was found and a methicillinresistant Staphylococcus aureus (MRSA) was cultured. After thorough debridement, girdlestone arthroplasty was done. The patient received specific intravenous antibiotics and after six weeks a total hip arthroplasty was done. In three years follow-up the patients presented with a fully functional hip without any signs of infection. Hip subluxation after a trochanteric fracture internal fixation is rare complication associated with high morbidity and mortality. Infection eradication holds a key for successful outcome in these cases.
\end{abstract}

Keywords: Infection; Neck of femur; DHS

\section{Introduction}

Intertrochanteric fractures of the proximal femur are extremely common, with an incidence of approximately 350 per 100,000 in the UK [1]. The incidence is expected to increase in the coming years, with an ageing western popu-

Manuscript accepted for publication August 12, 2010

${ }^{a}$ Hull Royal Infirmary, Hull, UK

${ }^{\mathrm{b}}$ Leeds Teaching Hospital NHS Trust, Leeds, UK

${ }^{\mathrm{c}}$ Corresponding author: Specialist Registrar Hull Royal Infirmary, Hull,

UK. Email: rohit.rambani@gmail.com

doi:10.4021/jmc31w lation resulting in a greater number of traumatic injuries in patients with osteoporotic bone.

The vast majority of intertrochanteric fractures are treated by surgical means. A number of different methods for surgical fixation are recognized, including intramedullary nail and hip screw devices, sliding compression screw and plate devices, and external fixators. The dynamic hip screw (DHS) has been in use since the 1950's and its use has become standard practice in the surgical treatment of intertrochanteric femoral fractures [2]. Furthermore, it has been shown to be superior to cephalomedullary devices due to smaller risk of intra and postoperative femoral fracture and subsequent reoperation, with no difference in clinical outcome or rate of technical complications [2].

Complications of DHS implantation for fixation of proximal femoral fractures include osteonecrosis of the femoral head, disassembly of the screw and plate, cutting out of the lag-screw from the femoral head, bleeding complications, hematoma, and wound infection. The incidence of wound infection following DHS fixation for extracapsular femoral neck fractures has been reported to be between 5 and $16 \%$, although the use of prophylactic antibiotics as standard has reduced this to $1 \%[3]$.

There have been a small number of reports of deep wound infection presenting with hip joint dislocation. Subluxation of the hip following internal fixation of the hip has also been reported a number of times. However, to our knowledge, a case of deep wound infection complicating internal fixation of an intertrochanteric femoral neck fracture, presenting as a painful subluxating hip joint, has not been reported.

\section{Case Report}

An 86-year-old female was referred by the GP to accident and emergency with difficulty in mobilizing and pain in the left hip three months after an uneventful fixation of extra capsular fracture of femoral neck with dynamic hip screw. She presented without fever, wound drainage, abscess formation or cellulitis. X-ray imaging performed revealed a left hip subluxation (Fig. 1 and 2). The patient had been discharged from the hospital nearly 2.5 months ago and had 


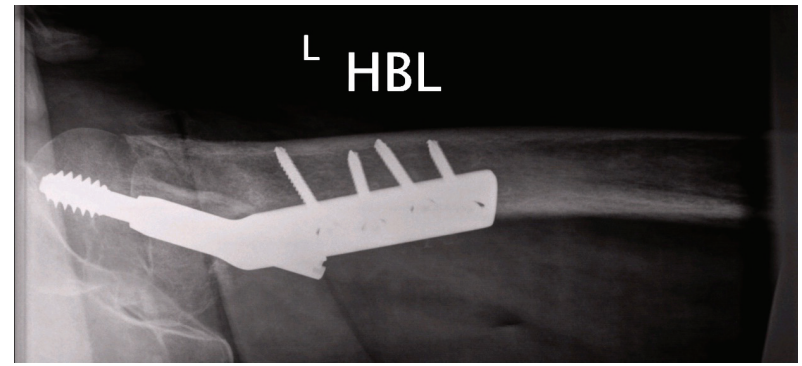

Figure 1. X-ray imaging revealed a left hip subluxation.

no postoperative complications and the fracture has united. Her medical history revealed hypertension and type II diabetes mellitus. She was afebrile at presentation and did not give any history of fever in the last three months. Laboratory tests revealed normal CRP, ESR and total Leucocytic count. Her physical examination and the rest of the laboratory tests (chest x-ray, urine analysis) were normal. The patient was listed for surgery to reduce the hip with closed reduction or open reduction. When closed reduction was unsuccessful, open reduction was done which showed deep wound infection with tissue inflammation and necrosis. The internal fixation was stable and the trochanteric fracture had healed.

Soft tissue debridement was done to remove all infected and nonviable tissue. Fluid found and specimens of infected deep soft tissues were sent for immediate Gram's stain, culture and antibiotic sensitivity tests. The Gram's stain revealed numerous polymorphonuclear cells. Since the fracture had healed, the hip joint was dislocated and inflamed with part of the femoral head eroded, an excisional arthroplasty was done and reaming sent for histopathology.

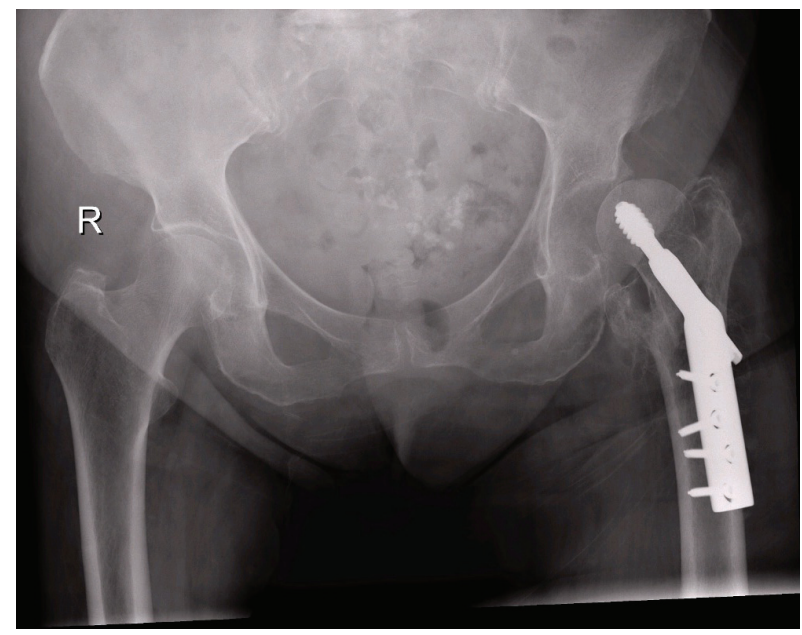

Figure 2. X-ray imaging revealed a left hip subluxation.

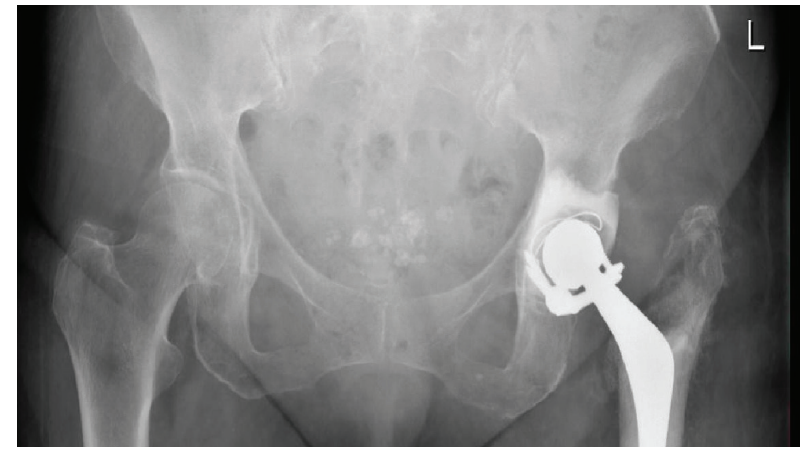

Figure 3. The second stage surgery was performed at this time with Constrained Cemented Total hip arthroplasty with antibiotic loaded cement.

A skeletal traction system from the tibial tuberosity was used to maintain muscle tension. The wound specimens' cultures recovered a methicillin-resistant Staphylococcus aureus (MRSA) sensitive to vancomycin. The patient was administered IV antibiotics for six weeks. The second stage surgery was performed at this time with Constrained Cemented Total hip arthroplasty with antibiotic loaded cement (Fig.3).

The patient had a postoperative period without complications. In the follow-up, three years after the arthroplasty, she can walk without pain or limp, the hip wound shows no signs of infection and the laboratory results are within normal values.

\section{Discussion}

This case is, to our knowledge, the first reported case of deep infection of the hip joint following dynamic hip screw fixation of a femoral neck fracture, presenting with subluxation of the hip. A number of authors have reported dislocation of the hip as the presenting feature of deep wound sepsis following internal fixation of the hip, but cases of hip subluxation as a presenting feature is extremely rare. Speed and Knight [4] first reported a septic dislocation following hip fixation in 1956. Melton et al [5] reported dislocation of the hip following fixation of a pertrochanteric fracture in a valgus position. There have been several reports of atraumatic dislocation of the hip joint following internal fixation, but these have not been associated with sepsis and presented with a dislocated joint as opposed to a subluxating joint [6].

Subluxation of the hip joint has been widely reported in patients with cerebral palsy, due to abnormal mechanical stresses on the hip joint from muscle spasticity [7]. A number of corrective and preventative procedures have been described for this problem including iliopsoas transfer with adductor tenotomy, proximal femoral resection and hip distraction with external fixation, rectus femoris tenotomy, and 
release or transposition of knee flexors. Hip joint subluxation has also been reported in other conditions affecting the hip such as myositis ossificans and synovial chondromatosis [7]. However, following internal fixation for a fracture of the proximal femur, the only recent report of hip joint subluxation is that of Kaneko et al [5], but this was not a presentation of deep wound sepsis.

Wound infection following internal fixation has now been reduced to approximately $1-2 \%$ with prophylactic antibiotic regimes [3]. In the United Kingdom, Edwards and colleagues conducted a large prospective single centre study of 3,686 patients with hip fractures, reporting an incidence of early deep wound infection of $1.2 \%$, and of superficial wound infection of $1.2 \%$. Interestingly, the authors reported that $71.3 \%$ of all infections were due to Staphyloccus Aureus, with Methicillin resistant strains accounting for $48.8 \%$ [8]. These results echoed those from a Finnish study which retrospectively reviewed over 2,000 hip fracture cases, reporting an incidence of deep infection of $1.3 \%$, with S. Aureus being the commenest causative organism. Furthermore, the authors demonstrated a significantly higher mortality risk with S. Aureus infection, compared to other organisms. It is clear that the implications of deep wound infection can be catastrophic for the patient in terms of quality of life and clinical outcome. In particular, deep infection of the hip joint has been shown to significantly affect functional outcome, prolong hospital stay and increase mortality by $10.4 \%$ [9]. Late infection of the hip following internal fixation is less well documented however. As with other reports in adults, there was a variable time to presentation postoperatively ranging from 7 to 24 months, and S. Aureus was the commonest causative organism.

A number of mechanisms for subluxation of the hip joint have been described. Moorman and colleagues [10] reported the mechanism of traumatic hip dislocation in their clinical and radiological study of American Football players. The authors reported that a fall onto a flexed and adducted hip was the commonest mechanism, with MRI and radiographic findings often showing a posterior lip acetabular fracture with disruption of the iliofemoral ligament. The mechanism for atraumatic subluxation of the hip due to infection is not clear however. It is likely that an inflammatory process due to infection of the joint capsule and surrounding ligaments, including the iliofemoral liagament, will cause generalized instability and progressive subluxation due to weight-bearing and repetitive minor trauma. In ankylosing spondylitis, hyperostosis and ossification within the acetabulum and ligamentum teres has been shown to cause superolateral joint space narrowing and subsequent lateral subluxation of the joint [11]. This effect of progressive lateral migration of the femoral head may have been responsible in the present case, due to infective effusion within the hip joint, accompanied by destruction of the capsule and surrounding soft tissues, pushing the femoral head laterally, resulting in similar su- perolateral joint space narrowing and subluxation.

The patient reported in the present study underwent a two-stage revision hip arthroplasty due to deep wound sepsis. A two-stage revision procedure, with antibiotic cement spacer and intravenous antimicrobial therapy, before the definitive procedure, was the prudent option in this case. The destruction of local soft tissues obviated the need for complete eradication of infection and inflammatory process, as ongoing infection and soft tissue destruction would be potentially catastrophic for the ongoing stability of the hip, as well as the functional outcome of the patient. This case highlights the difficulties presented by deep wound infection and the cost to both the patient and hospital services.

The present case report highlights the importance of keeping a high level of suspicion for deep infection in elderly patients with minimal clinical signs and symptoms of infection. Hip subluxation after a trochanteric fracture internal fixation is rare complication associated with high morbidity and mortality. Infection eradication holds a key for successful outcome in these cases.

\section{Consent}

Written informed consent was obtained from the patient for publication of this case report and accompanying images. A copy of the written consent is available for review by the Editor-in-Chief of this journal.

\section{Competing Interests}

The authors declare that they have no competing interests.

\section{References}

1. Holt G, Smith R, Duncan K, Hutchison JD, Reid D. Changes in population demographics and the future incidence of hip fracture. Injury 2009;40(7):722-726.

2. Anglen JO, Weinstein JN. Nail or plate fixation of intertrochanteric hip fractures: changing pattern of practice. A review of the American Board of Orthopaedic Surgery Database. J Bone Joint Surg Am 2008;90(4):700-707.

3. Bodoky A, Neff U, Heberer M, Harder F. Antibiotic prophylaxis with two doses of cephalosporin in patients managed with internal fixation for a fracture of the hip. $\mathrm{J}$ Bone Joint Surg Am 1993;75(1):61-65.

4. Speed JS, Knight RA. Postoperative infections following internal fixation for fractures of the hip. Ann Surg 1956;143(5):651-657; discussion, 657-659.

5. Melton JT, Yates P, Middleton RG. Dislocation of the hip following valgus fixation of unstable pertrochanteric fractures: A complication. Injury Extra 2006. 
6. Combalia A. Hip dislocation after internal fixation without infection. Can J Surg 1998;41(1):72.

7. Hardacker J, Mindell ER. Synovial chondromatosis with secondary subluxation of the hip. A case report. J Bone Joint Surg Am 1991;73(9):1405-1407.

8. Edwards C, Counsell A, Boulton C, Moran CG. Early infection after hip fracture surgery: risk factors, costs and outcome. J Bone Joint Surg Br 2008;90(6):770-777.

9. Partanen J, Syrjala H, Vahanikkila H, Jalovaara P. Impact of deep infection after hip fracture surgery on func- tion and mortality. J Hosp Infect 2006;62(1):44-49.

10. Moorman CT, 3rd, Warren RF, Hershman EB, Crowe JF, Potter HG, Barnes R, O’Brien SJ, et al. Traumatic posterior hip subluxation in American football. J Bone Joint Surg Am 2003;85-A(7):1190-1196.

11. Inoue K, Schichikawa K, Takenaka Y, Yamauchi E, Nishioka J, Hukuda S. Ossification of the ligamentum teres as a possible mechanism of lateral subluxation in coxopathy associated with ankylosing spinal hyperostosis. Ann Rheum Dis 1993;52(4):306-307. 